\title{
The stem cell niches in bone
}

\author{
Tong Yin ${ }^{1}$ and Linheng $\mathrm{Li}^{1,2}$ \\ ${ }^{1}$ Stowers Institute for Medical Research, Kansas City, Missouri, USA. ${ }^{2}$ Department of Pathology and Laboratory Medicine, \\ Kansas University Medical Center, Kansas City, Kansas, USA.
}

\begin{abstract}
The stem cell niche is composed of a specialized population of cells that plays an essential role in regulating adult stem cell self-renewal and differentiation. In adults, osteoblasts, responsible for osteogenesis, and hematopoietic cells, responsible for hematopoiesis, are closely associated in the bone marrow, suggesting a reciprocal relationship between the two. It was recently discovered that a subset of osteoblasts functions as a key component of the HSC niche (namely, the osteoblastic niche), controlling HSC numbers. HSCs interact not only with osteoblasts but also with other stromal cells, including endothelial cells. Sinusoidal endothelial cells in bone marrow have been revealed as an alternative HSC niche called the vascular niche. In this Review we compare the architecture of these 2 HSC niches in bone marrow. We also highlight the function of osteoblasts in maintaining a quiescent HSC microenvironment and the likely role of the vascular niche in regulating stem cell proliferation, differentiation, and mobilization. In addition, we focus on studies of animal models and in vitro assays that have provided direct insights into the actions of these osteoblastic and vascular niches, revealing central roles for numerous signaling and adhesion molecules. Many of the discoveries described herein may contribute to future clinical treatments for hematopoietic and bone-related disorders, including cancer.
\end{abstract}

\section{Introduction}

Bone and bone marrow. Bone plays an essential role in the structure, protection, and movement of the body. Mammalian bone consists of bone cells at different developmental stages (including preosteoblasts, osteoblasts, and osteocytes), collagen fibrils, and mineral deposits such as calcium and phosphate $(1,2)$. The bone cavity is filled with soft BM and blood vessels. The developing hematopoietic cells within the bone cavity are retained in the BM until they have matured and are released into the vascular system $(2,3)$. HSCs and their progeny are surrounded by stromal cells in BM (Figure 1). Mesenchymal stem cells (MSCs) also reside in the bone cavity and are proposed to give rise to the majority of marrow stromal cell lineages, including chondrocytes, osteoblasts, fibroblasts, adipocytes, endothelial cells, and myocytes, as demonstrated in vitro and partially in vivo (4-6). In addition, endothelial cells may also originate from hemangioblasts, progenitor cells that can give rise to both hematopoietic and endothelial cells during embryonic stage (7-9). However, thus far the location and lineage commitment of MSCs in vivo are much less characterized than those of HSCs. We do know that a close relationship exists between osteogenesis and hematopoiesis and that osteoblast cells play an important role not only in skeletal development but also in the regulation of hematopoiesis $(10,11)$

The HSC niche and associated stromal cells in bone marrow. A stem cell niche is a specific site in adult tissues where stem cells reside and undergo self-renewal and produce large numbers of progeny (differentiation). Structurally, the niche is formed by supporting cells that provide a microenvironment for stem cells as well as the signals emanating from the supporting cells (12-14). The concept of the HSC niche was first proposed by Schofield in 1978 to describe

Nonstandard abbreviations used: Ang-1, angiopoietin 1; BMP, bone morphogenic protein; $\mathrm{CaR}, \mathrm{Ca}^{2+}$-sensing receptor; HPC, hematopoietic progenitor cell; LT-HSC, long-term HSC; MSC, mesenchymal stem cell; OPG, osteoprotegerin; PTH, parathyroid hormone; PTHrP, PTH-related protein; RANKL, receptor activator of NF- $\mathrm{KB}$ ligand; SDF-1, stromal cell-derived factor 1; TK, herpesvirus thymidine kinase; VE-cadherin, vascular endothelial cadherin.

Conflict of interest: The authors have declared that no conflict of interest exists. Citation for this article: J. Clin. Invest. 116:1195-1201 (2006). doi:10.1172/JCI28568. the physiologically limited microenvironment in which stem cells reside (15). The niche hypothesis has been supported by a variety of coculture experiments in vitro (16-21). Although HSC homing, or return, to the endosteal (inner) surface of bone is revealed by homing assays (22), the exact location, cellular composition, and associated molecular signals of the HSC niche remained elusive until recently. In mammals, BM composed mainly of hematopoietic cells is encased by the bone structure. A portion of these hematopoietic cells can be found next to the endosteal bone surface, which is lined primarily by osteoblasts. This anatomic arrangement suggests reciprocal communication between the 2 cell types and a potential role for osteoblasts (responsible for bone growth) in regulating HSCs (responsible for blood formation) (10). This notion is supported by the observation that osteoblasts are known to produce a variety of hematopoietic growth factors $(23,24)$, including the receptor activator of NF-KB ligand (RANKL), which plays an essential role in induction of osteoclast differentiation (25). Of note, osteoclasts are generated from hematopoietic precursor cells and are essential for bone resorption. On the other hand, osteopetrotic (op/op) mice, which lack M-CSF, demonstrate a loss of progenitor cells for both macrophages and osteoclasts. The resulting osteopetrosis arises due to a lack of osteoclasts (26). Accumulated evidence supports the model that HSCs residing next to the endosteal bone surface produce progenitors that migrate to blood vessels at the center of the BM cavity as they mature and differentiate $(22,27,28)$. A role for osteoblasts in supporting HSC growth was suggested through in vitro coculture experiments (29, 30 ), and several studies simultaneously identified osteoblastic cells as a key component of the HSC niche using genetic mutant mouse models (31-33). These results are consistent with the observation that conditional ablation of osteoblasts leads to depletion of hematopoietic cells, including HSCs (34), and demonstrate the role of osteoblasts in supporting HSCs.

Some hematopoietic progenitor cells (HPCs) appear to contact the sinusoidal endothelium in $\operatorname{BM}(21,35)$. Furthermore, in vivo ablation of endothelial cells using anti-vascular endothelial cadherin (anti-VE-cadherin) antibody leads to hematopoietic failure (36), similar to results obtained from osteoblast deple- 


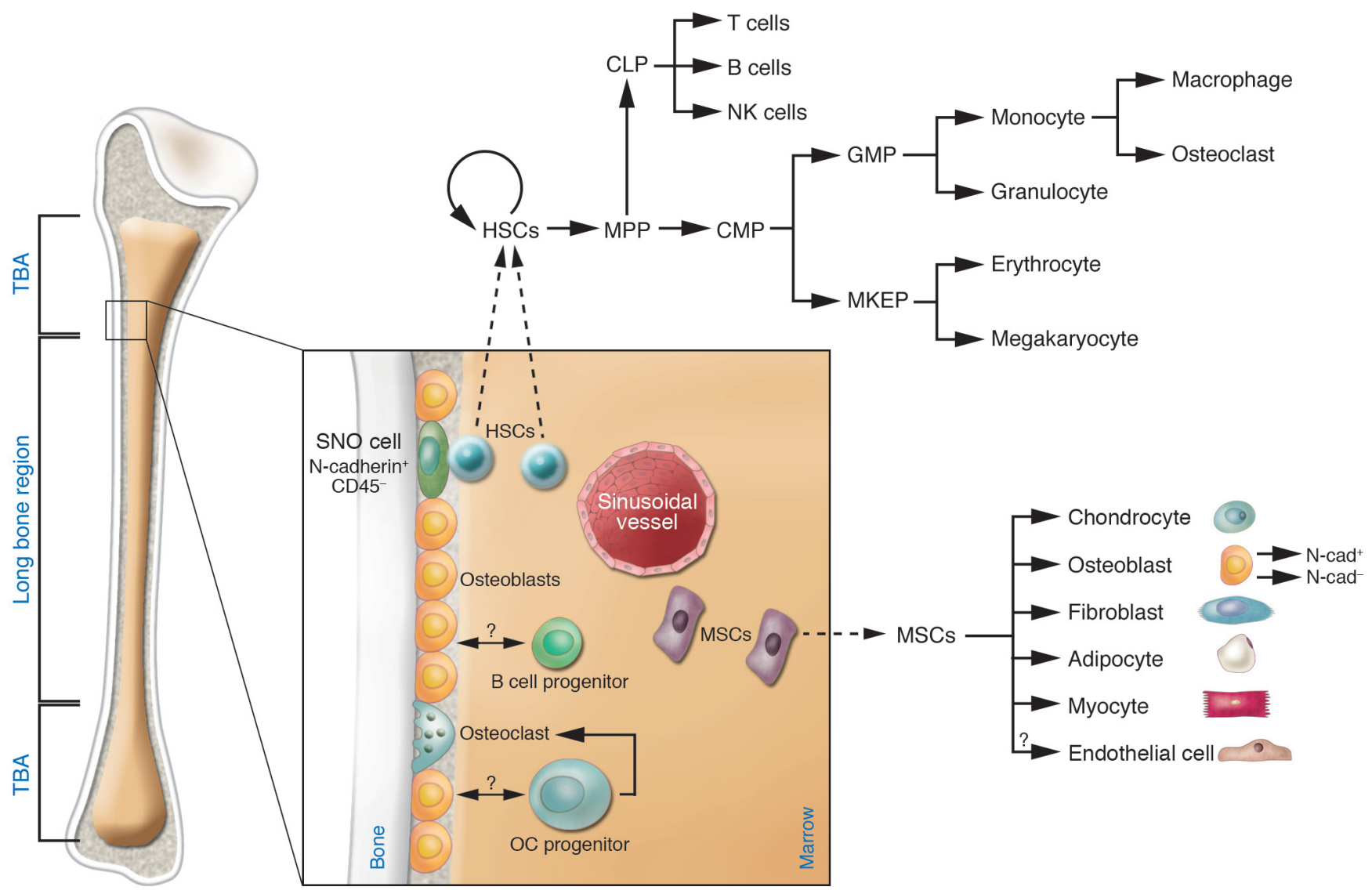

Figure 1

Hematopoiesis of bone cells and marrow stromal cells. In the bone marrow HSCs reside either next to osteoblasts on the endosteal niche or adjacent to endothelial cells of sinusoidal vessels. Upon each division, 1 daughter cell leaves the bone to proliferate and differentiate into various lineages. HSCs and their progeny are surrounded by stromal cells derived from MSCs, which also reside in the bone cavity. MSCs give rise to chondrocytes, osteoblasts, fibroblasts, adipocytes, endothelium, and myocytes. Osteoblasts (expressing M-CSF, RANKL, and OPG and producing IL-7) may regulate lymphoid (expressing IL-7R) and osteoclast (derived from monocytes, expressing the M-CSF ligand c-Fms and RANK) cell development. CMP, common myeloid progenitor; CLP, common lymphoid progenitor; GMP, granulocyte/macrophage progenitor; MKEP, megakaryocyte erythroid progenitor; MPP, multipotent progenitor stem cell; OC, osteoclast progenitor; SNO cell, spindle-shaped $\mathrm{N}$-cadherin+CD45- osteoblastic cell; TBA, trabecular bone area.

tion experiments. Recently, a combination of surface markers of the signaling lymphocyte activation molecule (SLAM) family $\left(\mathrm{CD} 150^{+} \mathrm{CD} 48^{-} \mathrm{CD} 41^{-}\right)$was shown to be an HSC identifier. HSCs expressing SLAM were found adjacent to the bone surface as well as to endothelial cells in both BM and spleen (37). The interaction of HSCs with the sinusoidal endothelium suggests that endothelial cells create an alternative niche. To distinguish it from the osteoblastic niche, the endothelial cell-containing vascular zone in BM is termed the vascular niche (37-39).

In summary, at least 2 distinct niches supporting HSCs have been identified in BM: the osteoblastic niche and the vascular niche. In this Review, we will compare the cellular composition of these 2 niches in bone and discuss their potential functions and relationship.

\section{Homeostatic regulation of stem cells by the niche}

The ability of adult stem cells to both self-renew and differentiate is critical for tissue homeostasis. The stem cell population would become depleted if cell differentiation overwhelmed self-renewal. Similarly, unchecked stem cell self-renewal would expand the stem cell population excessively, risking tumorigenesis (40). An impor- tant function of the stem cell niche, therefore, is to regulate the balance between cellular self-renewal and differentiation. One mechanism that ensures this balance is the control of asymmet$\mathrm{ric} /$ symmetric stem cell division. Asymmetric division means that stem cells divide into 2 daughter cells; one daughter cell remains in the niche as a stem cell and the other leaves the niche to produce a large number of progeny. Symmetric division means that stem cells divide into 2 identical daughter cells, both remaining in the niche as stem cells. Switching between symmetric and asymmetric division can occur in multiple stem cells that occupy the same niche under different physiological conditions $(41,42)$. In Drosophila germ stem cells, cell division is asymmetric or symmetric depending on whether the orientation of the mitotic spindle is perpendicular or parallel to the interface between the stem cell and its niche. The mechanisms underlying the establishment of cell polarity and spindle orientation are complex and, in some cases, appear to involve overlapping subsets of factors including adenomatous polyposis coli (APC), centrosomin, and adherens junction-related cadherins and catenins (42-44). Whether stem cells normally undergo asymmetric division in mammals as they do in invertebrates is yet to 
be determined. The impact of the niche size on controlling the number of stem cells supports the model that stem cells, at least in the hematopoietic system, most likely execute asymmetric division under normal physiological conditions $(31,32)$.

\section{The role of the osteoblastic niche in supporting HSC maintenance}

There are 2 classifications of HSCs, namely, long-term and shortterm. Long-term HSCs (LT-HSCs) are capable of contributing to hematopoiesis for months or even a lifetime. Short-term HSCs (ST-HSCs) have a reconstitution ability that is limited to several weeks $(45,46)$. LT-HSCs are maintained primarily as quiescent or slow-cycling cells, while ST-HSCs are actively cycling (47). Data from conditional inactivation of bone morphogenic protein (BMP) receptor type IA (BMPRIA) mice reveal that an increase in the number of spindle-shaped $\mathrm{N}$-cadherin ${ }^{+} \mathrm{CD} 45^{-}$osteoblastic (SNO) cells, a subset of osteoblastic lining cells, in the trabecular bone area correlates with an increase in the number of LT-HSCs, suggesting that SNO cells function as a key component of the niche to support HSCs (31) (Figure 1). Similarly, overexpression of parathyroid hormone $(\mathrm{PTH})$ and $\mathrm{PTH}$-related protein (PTHrP) receptor leads to increased osteoblast numbers, resulting in a parallel increase in the number of HSCs in the $\mathrm{PTH} / \mathrm{PTHrP}$ receptor (PPR) transgenic mouse model (32). Both of these studies point to the dependence of LT-HSCs on the osteoblastic niche. It appears that the ability of the osteoblastic niche to retain stem cells in a quiescent state is an important mechanism in maintaining sufficient stem cells (33).

Additional evidence supports a role for the osteoblastic niche in supporting and maintaining HSCs. Visnjic and colleagues have demonstrated that HSCs, and, in fact, the hematopoietic system, rely on osteoblasts for support (34). This result came from studies of transgenic mice that specifically express the herpesvirus thymidine kinase (TK) gene in developing osteoblasts under the control of the collagen $\alpha_{\text {I }}$ promoter (Col2.3 $\left.\Delta \mathrm{TK}\right)$. The advantage of the Col2.3 $\Delta \mathrm{TK}$ mice is the ability to inducibly ablate osteoblasts with ganciclovir (GCV), which triggers TK function. Loss of osteoblasts in GCV-treated Col2.3 $\Delta$ TK mice led to substantial decreases in the number of lymphoid, erythroid, and myeloid progenitors (including osteoclast progenitors) in the BM, followed by a decrease in the absolute number of HSCs (although the percentage of HSCs appeared only slightly changed) and reduced BM cellularity. After withdrawal of GCV, osteoblasts reappeared in the bone, as did hematopoiesis in the BM. These observations further support the role of osteoblasts in the maintenance of HSCs and regulation of hematopoiesis, although the authors did not perform functional assays to test HSCs in this model. In another study osteoblasts were shown to facilitate engraftment of HSCs in an allogeneic environment. After purified osteoblasts were cotransplanted with marrow stem cells into allogeneic mouse strains, the transplanted recipient mice demonstrated excellent long-term survival, absence of disease, and complete engraftment by the donor cells. It appeared that the HPCs could engraft lethally irradiated mice, but these cells could not cross the MHC antigen barrier in the absence of cotransplanted osteoblasts (48).

The role of the vascular niche in supporting proliferation, differentiation, and transendothelial migration of HSCs

Hematopoiesis and vascularization occur concurrently during development. In fact, HSCs and endothelial cells are derived from the same progenitor cells (termed hemangioblasts) at the embry- onic stage and are closely related to the ontogeny of hematopoiesis that occurs in the yolk sac, aorta-gonad-mesonephros, placenta, fetal liver, spleen, and adult BM $(7,8,49)$. Indeed, HSCs expressing SLAM markers were detected on the osteoblastic surface of trabecular bone as well as adjacent to sinusoidal endothelial cells (37). This is consistent with another study in which explanted endothelial cells were able to maintain HSCs in culture (21). Together these observations support the model that HSCs may use either osteoblasts or endothelial cells as their niche under different circumstances (39). The discovery that sinusoid endothelial cells have the potential to act as part of an alternative HSC niche together with the known osteoblastic niche support a previously proposed model in which the osteoblastic niche provides a quiescent microenvironment and the vascular niche promotes proliferation and further differentiation (38). This model is exemplified in studies of thrombopoietin mutant $\left(\mathrm{TPO}^{-/-}\right)$mice, which revealed that reconstitution of thrombopoiesis under stress involves recruitment of HSCs to the sinusoid endothelial surface. This action involves MMP-9, which mediates release of soluble Kit ligand (sKitL). In this study HSCs left the osteoblastic niche and translocated to the vascular niche, where they differentiated into megakaryocyte progenitors for further megakaryocyte maturation as well as platelet release $(36,38)$. Other studies also support a more prominent role for osteoblasts in maintaining HSC quiescence and suggest that the sinusoidal endothelium promotes proliferation and differentiation of stem and progenitor cells by providing a more nutrient-rich microenvironment, with higher concentrations of oxygen and growth factors and in which mature blood cells are ultimately released into the peripheral circulation (Figure 2) $(38,50)$.

A second plausible function for the vascular niche, discussed later in this Review, is to assist HSCs in transendothelial migration, important during both homing and mobilization $(51,52)$. A third function of the vascular niche is assigned to cells located outside of the BM medullary space, such as in the spleen. These cells might serve to replace the BM niche when the BM niche is under stress, such as with marrow suppression (37). Splenomegaly concomitant with extramedullary hematopoiesis is a common phenomenon in both humans and genetically mutant mice that suffer from BM hematopoietic failure. Based on these observations it is reasonable to hypothesize that endothelial cells may also function as a "backup" niche to support HSC expansion and produce a large number of progeny for hematopoiesis when the $\mathrm{BM}$ is under stress.

\section{The relationship between the osteoblastic and vascular niches}

It is well known that HSC circulation involves HSCs leaving the BM, entering the vascular system (mobilization), and returning to the $\mathrm{BM}$ (homing). However, the underlying physiological function of these events is not well understood (53). The BM vascular structure provides a barrier between the hematopoietic compartment and the peripheral circulation. Most primitive HSCs remain physiologically quiescent within the BM niche; however, a portion of HSCs leave this resting pool and initiate the process of mobilization $(28,33$, 39). There are some controversial reports suggesting that mobilized HSCs may be more quiescent than those that remain in the $\mathrm{BM}$. Evidence to support this notion comes mainly from a study in which the CD $34^{+}$marker was used to identify HSCs and HPCs (54). Since the population of $\mathrm{CD} 34^{+}$cells included numerous progenitor cells, the comparison of the cell cycle profile of the CD $34^{+}$cells residing in $\mathrm{BM}$ and in circulating peripheral blood in this study 




Figure 2

The osteoblastic and vascular niches in bone. Under normal physiological conditions, HSCs reside in either the osteoblastic or vascular niche. A portion of HSC daughter cells, in response to changes in levels of SDF-1 in the BM, will leave the niche and begin to mobilize and circulate. HSC homing is the reverse of mobilization, occurring in response to higher levels of SDF-1 in the BM. The osteoblastic niche may provide a quiescent microenvironment for HSC maintenance. In contrast, the vascular niche facilitates HSC transendothelial migration during mobilization or homing and may favor HSC proliferation and further differentiation. The process of recruiting HPCs to the vascular niche may depend on endothelium-derived FGF-4 and SDF-1. Higher FGF-4 and oxygen concentration gradients as the cells progress from the osteoblastic niche to the vascular niche might play a role in recruitment, proliferation, and differentiation of HSCs/HPCs. Under stress such as thrombocytopenia, SDF-1 and VEGF activate MMP-9, which converts membrane-associated Kit ligand into soluble Kit ligand (sKitL) and in turn promotes HSCs entry into the cell cycle, mobilization to the vascular niche, and differentiation (34).

could not reflect the most primitive HSCs. Indeed, Weissman and colleagues have definitively shown in mice that mobilized HSCs are more proliferative than LT-HSCs in BM by comparing highly purified BM LT-HSCs and mobilized HSCs (47).

Both osteoblastic and vascular niches may play important roles in regulating HSC mobilization. This includes HSCs leaving the osteoblastic niche, mobilizing to the vascular niche, entering the blood vessel through the endothelial cells, and circulating in the vascular system, where HSCs are in constant contact with endothelial cells (Figure 2). Homing of stem cells is simply the reverse of this process, with HSCs returning from the peripheral circulation, undergoing transendothelial migration to the vascular niche, and finally coming back to the osteoblastic niche $(51,52)$.

\section{Molecules that mediate signaling and adhesive interactions between stem cells and their niches}

Multiple signaling and adhesion molecules are involved in stem cell-niche interactions, contributing diverse characteristics to each niche's function. Well-studied signaling molecules involved in niche regulation include $\mathrm{SCF} / \mathrm{c}$-Kit, Jagged/Notch, angiopoietin-1/ Tie2 (Ang-1/Tie2), and $\mathrm{Ca}^{2+}$-sensing receptor $(\mathrm{CaR})(28,32,33$, $55,56)$. This section will cover these as well as other molecules, including Wnt, BMP, TPO, IL-3, and IL-6, known to play roles in stem cell regulation. It is presently unclear whether all of the latter molecules are present in the niches.
SCF signaling through its receptor, c-Kit, has been well characterized in promoting both proliferation and survival of HSCs. Loss of SCF from supporting cells in $s l / s l$ mice, or loss of the receptor in HSCs in $W / W$ mice, leads to hematopoietic failure, indicating essential roles for these molecules in niche function (57). These results are supported by more recent work revealing a direct role for $\mathrm{SCF} / \mathrm{c}-\mathrm{Kit}$ signaling in control of HSC activation and release from the niche (28).

Notch is expressed in primitive HSCs, while the Notch ligand Jag1 is expressed by mouse osteoblasts and bone marrow stromal cells. Activation of the PPR can increase osteoblastic cell numbers concomitant with enhanced Jag1/Notch activity, resulting in expansion of the HSC population (32). This observation is consistent with previous reports that activation of Notch signaling is able to maintain undifferentiated stem and progenitor cells and expand the HSC/HPC pools $(58,59)$.

Ang-1 is expressed in osteoblasts, while Tie2, a tyrosine kinase receptor, is expressed in HSCs and endothelial cells. Ang-1 enhances the ability of HSCs to become quiescent and can induce adhesion to osteoblastic cells through Tie2 (33).

Recently, CaR was found to facilitate retention of HSCs on the endosteal bone surface. Deficiency of CaR in knockout mice leads to release of HSCs into the bloodstream. Interestingly, although CaR deficiency permitted HSC homing to BM in these animals, the cells were unable to remain localized to the osteoblastic niche (55). 
BMP and Wnt signaling pathways are also involved in stem cell regulation. BMP-4 is expressed in osteoblastic cells (J. Zhang and L. Li, unpublished observations), but the type of BMP receptor expressed in HSCs is unknown (12). Wnt signals are important for stem cell self-renewal $(60,61)$, but the identity of the niche (osteoblastic or vascular) that expresses any of numerous canonical and noncanonical Wnt molecules is unknown. The same is true for FGF and hedgehog, both of which affect HSC behavior in vitro $(62,63)$. However, whether and where these factors are present as niche signals remain unresolved. In addition, well-documented studies have shown that IL-3 or IL- 6 combined with TPO signaling can influence stem cell proliferation and differentiation $(64,65)$. However, details regarding whether they are present specifically within the osteoblastic or vascular niches are unknown.

\section{Adhesion molecules in the stem cell niches}

The adhesion molecules important in niche function include $\mathrm{N}$-cadherin/ $\beta$-catenin, VCAM/integrin, and osteopontin $/ \beta_{1}$ integrin (OPN $/ \beta_{1}$ integrin) $(12,66,67)$. They may play a role either for the attachment of stem cells to the niche or for migration of stem cells. Two adherens junction molecules, $\mathrm{N}$-cadherin and $\beta$-catenin, are asymmetrically localized at the interface between HSCs and the osteoblastic niche (31). The essential roles of cadherins and $\beta$-catenin in supporting germ-line stem cells and determining the asymmetric division and orientation of germ-line stem cells in Drosophila have been reported $(44,68)$. The specialized distribution of the adhesion complex composed of $\mathrm{N}$-cadherin and $\beta$-catenin suggests a potential role for these molecules in facilitating anchoring of HSCs to the osteoblastic niche as well as their involvement in regulating asymmetric division of HSCs (6). The precise functions of these adhesion molecules in this regard are yet to be determined. The role of integrins in mediating HSC migration and homing has been shown by blocking integrin function with anti-integrin antibody (69). Recently, OPN was found to contribute to HSC transmarrow migration toward the endosteal region through its interaction with $\beta_{1}$ integrin $(66,67)$.

Accumulated evidence suggests that coordination between cellcycle control and adhesion of a stem cell to its niche is required for the dynamic regulation of stem cells. This is exemplified in studies of c-Myc by Wilson and colleagues, who demonstrated that c-Myc is involved in controlling the balance between quiescence for maintenance and proliferation for further differentiation of stem cells by regulating the interaction between HSCs and their niches $(70,71)$. In response to mitogens (such as Wnt and $\mathrm{c}-\mathrm{Kit}$ ), c-Myc upregulation is required to release HSCs from the stem cell niche. Similarly, c-Myc expression is repressed by antiproliferative signaling factors such as TGF- $\beta$. Conditional elimination of c-Myc in BM results in severe cytopenia (insufficient supply of progenitor cells for differentiation) and accumulation of HSCs with upregulation of a number of adhesion molecules including $\mathrm{N}$-cadherin and integrins. Accordingly, forced c-Myc expression in HSCs has been shown to repress expression of $\mathrm{N}$-cadherin and integrins. Upon stem cell division, daughter cells expressing low levels of c-Myc are retained in the niche in the quiescent state due to upregulation of $\mathrm{N}$-cadherin and integrins. In contrast, high levels of c-Myc expression in remaining daughter cells, along with downregulation of cell adhesion molecules, causes these daughter cells to leave the niche for further proliferation and differentiation (70).

\section{The vascular niche}

The vascular niche was only recently identified $(28,37)$. As a result, the associated molecules involved in regulating HSCs within this niche remain largely unidentified. However, studies regarding recruitment of HPCs to the endothelial surface provide important insights into understanding HSC behavior as it relates to the vascular niche. Recruitment of HPCs to the vascular niche depends on FGF-4 and chemokines such as stromal cell-derived factor 1 (SDF-1; also called CXCL12) $(36,38)$. FGF-4 augments adhesion of megakaryocyte progenitors to the vascular niche, while SDF- 1 is a potent chemotactic factor for the transendothelial migration of CD $41^{+}$megakaryocytes. FGF-4-stimulated megakaryocyte adhesion or SDF-1-induced megakaryocyte migration can be blocked by chemical inhibitors of signal transduction pathways such as PI3K, PKC, and p38 MAPK, suggesting that multiple downstream signaling cascades are involved. FGF-4 and SDF-1 enhance the expression of the adhesion molecule very late antigen 4 (VLA-4) on megakaryocytes as well as VCAM-1 on endothelial cells. VEcadherin is essential for both proper assembly and mediation of adhesion of megakaryocyte progenitors to the vascular niche for survival and further maturation. Blocking VE-cadherin by antibodies results in impaired thrombopoiesis (36).

HSCs also express FGFR1, -2, and -3, and FGF has been shown to be able to stimulate HSC self-renewal and proliferation in vitro (63). Therefore it is conceivable that the FGF signals emanating from the vascular niche may play roles in recruiting HSCs/HPCs by forming a gradient from the osteoblastic niche (lower FGF expression) to the vascular niche (higher FGF expression) (38). This proposed regulatory role of FGF is consistent with its known ability to induce cell migration during embryonic development (72). HSCs also express CXCR4 and integrins, and the related functions of these molecules will be discussed below.

\section{Chemokines and other molecules involved in HSC/HPC homing and mobilization}

The ability of HSCs to mobilize and then return, or home, to the niche relies on specific molecular recognition, cell-cell adhesion/ disengagement, transendothelial migration, and finally anchoring to the BM niche $(6,51,52,73)$. The chemokine SDF- 1 and its receptor CXCR4 $(51,74,75)$ as well as underlying signaling pathways, including the Rac family molecules, regulate HSC mobilization and homing but also play a role in cell survival and proliferation $(51,76)$. Endothelial cells, osteoblasts, and other stromal cells constitutively express SDF-1, while HSCs express CXCR4 $(77,78)$. SDF-1 generated from endothelial cells induces HSCs to undergo transendothelial migration mediated by E- and P-selectins (79). Activation of adhesion molecules such as VLA-4 and leukocyte function antigen-1 (LFA-1) is also required for this process and the subsequent migration in BM toward the osteoblast surface (38). Likewise, high levels of SDF-1 on the surface of osteoblasts attract HSCs to return home to the osteoblast niche. The Rho GTPases Rac1 and Rac2, in response to SDF-1 signals, are also involved in regulating HSC mobilization and homing (80).

G-CSF induces HSC and progenitor cell mobilization and is widely used clinically during stem cell-based transplantation procedures. Recent studies revealed that the mechanism involved is primarily a decrease of SDF-1 in osteoblasts, with an increase of SDF-1 in peripheral circulation after G-CSF treatment (81). G-CSF also induces proteolytic enzymes such as elastase, cathepsin $G$, MMP-2, and MMP-9 (required for cells to penetrate the endothe- 
lium), which inactivates SDF-1 through cleavage of its $\mathrm{NH}_{2}$-terminal signal sequence (82). In addition, G-CSF could regulate SDF-1 expression in BM at the transcriptional level $(83,84)$. During stem cell homing HSCs expressing CXCR4 are attracted to the osteoblastic niche, which expresses high levels of SDF-1 $(36,38)$, and the adherens complex formed by $\mathrm{N}$-cadherin and $\beta$-catenin may play a crucial role for anchoring HSCs to the osteoblastic niche $(6,31)$.

\section{Not all osteoblasts function as part of the HSC niche}

Osteoblasts are heterogeneous and include the spindle-shaped endosteal lining cells and the oval-shaped cells that are the direct precursors of osteocytes. Among the osteoblastic lining cells, only a subset of osteoblasts $\left(\mathrm{N}\right.$-cadherin $\left.{ }^{+}\right)$interacts with HSCs (31). This demonstration is consistent with the observation that the niche size is tightly controlled in vivo in order to maintain a constant number of HSCs. In addition to supporting HSCs, it is possible that different subtypes of osteoblasts may play a role in regulating some, if not all, HPCs (Figure 1).

Signaling molecules between osteoblasts and osteoclast precursors are required for osteoclast maturation $(75,85,86)$. Osteoblasts and stromal cells express RANKL, M-CSF, and osteoprotegerin (OPG), while early osteoclast precursors express c-Fms (M-CSF receptor) and RANK (a receptor for RANKL) (85). RANKL and M-CSF stimulate osteoclast differentiation. However, OPG, as an inhibitor of RANKL, competes with RANKL for RANK (87). Animals lacking OPG have accelerated osteoclast development and develop severe osteoporosis, in which bone resorption exceeds bone growth (88).

There is also a close relationship between osteoblasts and lymphocyte development and differentiation $(89,90)$. Signaling molecules and receptor/ligand-based regulation of osteoblast and lymphoid progenitors are indispensable for B cell lymphopoiesis and maturation. As an essential growth factor for B cell commitment and development, IL-7 is produced by osteoblasts and other stromal cells while its receptor, IL-7R, is expressed in B progenitor cells. Deficiency of either IL-7 or IL-7R results in severe defects in B cell develop- ment $(91,92)$. All of these observations suggest that osteoblasts play a role in facilitating $\mathrm{B}$ cell progenitor commitment and development by providing stimulating factors such as IL-7 $\alpha$. The question arises as to whether this regulation is dependent on cell-cell interactions. The fact that a portion of the early lymphoid progenitor (ELP) cells, identified as a RAG-GFP ${ }^{+}$population (in which GFP expression is driven by the RAG1 promoter), are located on the endosteal surface in BM suggests that ELP cells may directly interact with osteoblasts (93). These findings spark the question of whether HSCs and other types of progenitors, such as B cell progenitors, share the same niche or use different subsets of osteoblasts.

\section{Conclusion}

Stem cell behavior is regulated by environmental (niche) signals and intrinsic programs. In this Review we have summarized the cellular architecture of the 2 niches in BM and discussed their potential functions. We have also discussed the important signals generated by these niches for their known and putative functions in regulation of HSC self-renewal, proliferation, differentiation, mobilization, and homing. This information may shed light on complicated regulation of HSCs in fulfilling different physiological requirements. The coordination between the osteoblastic niche and the vascular niche under different circumstances is provocative and merits future investigation. Finally, understanding the interaction between stem cells and their natural partners will substantially benefit therapeutic approaches to human diseases including hematopoietic and bone-related disorders.

\section{Acknowledgments}

We appreciate proofreading and editing by D. di Natale. We apologize to those whose papers are not cited here due to space limitations.

Address correspondence to: Linheng Li, Stowers Institute for Medical Research, 1000 East 50th Street, Kansas City, Missouri 64110, USA. Phone: (816) 926-4081; Fax: (816) 926-2023; E-mail: lil@stowers-institute.org.
1. Boskey, A.L., and Posner, A.S. 1984. Bone structure, composition, and mineralization. Orthop. Clin. North Am. 15:597-612.

2. Meghji, S. 1992. Bone remodelling. Br. Dent. J. 172:235-242.

3. Fliedner, T.M. 1998. The role of blood stem cells in hematopoietic cell renewal. Stem Cells. 16:361-374.

4. Wang, X., et al. 2005. Characterization of mesenchymal stem cells isolated from mouse fetal bone marrow. Stem Cells. doi:10.1634/stemcells. 2005-0219.

5. Short, B., Brouard, N., Occhiodoro-Scott, T., Ramakrishnan, A., and Simmons, P.J. 2003. Mesenchymal stem cells. Arch. Med. Res. 34:565-571.

6. Muguruma, Y., et al. 2006. Reconstitution of the functional human hematopoietic microenvironment derived from human mesenchymal stem cells in the murine bone marrow compartment. Blood. 107:1878-1887.

7. Hristov, M., and Weber, C. 2004. Endothelial progenitor cells: characterization, pathophysiology, and possible clinical relevance. J. Cell. Mol. Med. 8:498-508.

8. Lacaud, G., Robertson, S., Palis, J., Kennedy, M., and Keller, G. 2001. Regulation of hemangioblast development. Ann. N. Y. Acad. Sci. 938:96-107; discussion 108.

9. Robertson, S., Kennedy, M., and Keller, G. 1999. Hematopoietic commitment during embryogenesis. Ann. N. Y. Acad. Sci. 872:9-15; discussion 15-16.
10. Taichman, R.S. 2005. Blood and bone: two tissues whose fates are intertwined to create the hematopoietic stem-cell niche. Blood. 105:2631-2639.

11. Moore, K.A. 2004. Recent advances in defining the hematopoietic stem cell niche. Curr. Opin. Hematol. 11:107-111.

12. Li, L., and Xie, T. 2005. Stem cell niche: structure and function. Annu. Rev. Cell Dev. Biol. 21:605-631.

13. Spradling, A., Drummond-Barbosa, D., and Kai, T. 2001. Stem cells find their niche. Nature. 414:98-104.

14. Lin, H. 2002. The stem-cell niche theory: lessons from flies. Nat. Rev. Genet. 3:931-940.

15. Schofield, R. 1978. The relationship between the spleen colony-forming cell and the haemopoietic stem cell. Blood Cells. 4:7-25.

16. Dexter, T.M., Moore, M.A., and Sheridan, A.P. 1977. Maintenance of hemopoietic stem cells and production of differentiated progeny in allogeneic and semiallogeneic bone marrow chimeras in vitro. J. Exp. Med. 145:1612-1616.

17. Moore, K.A., Ema, H., and Lemischka, I.R. 1997. In vitro maintenance of highly purified, transplantable hematopoietic stem cells. Blood. 89:4337-4347.

18. Rios, M., and Williams, D.A. 1990. Systematic analysis of the ability of stromal cell lines derived from different murine adult tissues to support maintenance of hematopoietic stem cells in vitro. J. Cell. Physiol.145:434-443.

19. Roecklein, B.A., and Torok-Storb, B. 1995. Functionally distinct human marrow stromal cell lines immortalized by transduction with the human papiloma virus E6/E7 genes. Blood. 85:997-1005.

20. Sitnicka, E., Ruscetti, F.W., Priestley, G.V., Wolf, N.S., and Bartelmez, S.H. 1996. Transforming growth factor beta 1 directly and reversibly inhibits the initial cell divisions of long-term repopulating hematopoietic stem cells. Blood. 88:82-88.

21. Li, W., Johnson, S.A., Shelley, W.C., and Yoder, M.C. 2004. Hematopoietic stem cell repopulating ability can be maintained in vitro by some primary endothelial cells. Exp. Hematol. 32:1226-1237.

22. Nilsson, S.K., Johnston, H.M., and Coverdale, J.A. 2001. Spatial localization of transplanted hemopoietic stem cells: inferences for the localization of stem cell niches. Blood. 97:2293-2299.

23. Taichman, R.S., Reilly, M.J., and Emerson, S.G. 1996. Human osteoblasts support human hematopoietic progenitor cells in vitro bone marrow cultures. Blood. 87:518-524.

24. Taichman, R., Reilly, M., Verma, R., Ehrenman, K., and Emerson, S. 2001. Hepatocyte growth factor is secreted by osteoblasts and cooperatively permits the survival of haematopoietic progenitors. $\mathrm{Br}$. J. Haematol. 112:438-448.

25. Udagawa, N., et al. 1999. Osteoblasts/stromal cells stimulate osteoclast activation through expression of osteoclast differentiation factor/RANKL but not macrophage colony-stimulating factor: receptor activator of NF-kappa B ligand. Bone. 25:517-523.

26. Begg, S.K., et al. 1993. Delayed hematopoietic 
development in osteopetrotic (op/op) mice. J. Exp. Med. 177:237-242.

27. Gong, J.K. 1978. Endosteal marrow: a rich source of hematopoietic stem cells. Science. 199:1443-1445.

28. Heissig, B., et al. 2002. Recruitment of stem and progenitor cells from the bone marrow niche requires MMP-9 mediated release of kit-ligand. Cell. 109:625-637.

29. Taichman, R.S., and Emerson, S.G. 1994. Human osteoblasts support hematopoiesis through the production of granulocyte colony-stimulating factor. J. Exp. Med. 179:1677-1682.

30. Taichman, R.S., Reilly, M.J., and Emerson, S.G. 2000. The hematopoietic microenvironment: osteoblasts and the hematopoietic microenvironment. Hematology. 4:421-426.

31. Zhang, J., et al. 2003. Identification of the haematopoietic stem cell niche and control of the niche size. Nature. 425:836-841.

32. Calvi, L.M., et al. 2003. Osteoblastic cells regulate the haematopoietic stem cell niche. Nature. 425:841-846

33. Arai, F., et al. 2004. Tie2/angiopoietin-1 signaling regulates hematopoietic stem cell quiescence in the bone marrow niche. Cell. 118:149-161.

34. Visnjic, D., et al. 2004. Hematopoiesis is severely altered in mice with an induced osteoblast deficiency. Blood. 103:3258-3264.

35. Cardier, J.E., and Barbera-Guillem, E. 1997. Extramedullary hematopoiesis in the adult mouse liver is associated with specific hepatic sinusoidal endothelial cells. Hepatology. 26:165-175

36. Avecilla, S.T., et al. 2004. Chemokine-mediated interaction of hematopoietic progenitors with the bone marrow vascular niche is required for thrombopoiesis. Nat. Med. 10:64-71.

37. Kiel, M.J., Yilmaz, O.H., Iwashita, T., Terhorst, C., and Morrison, S.J. 2005. SLAM family receptors distinguish hematopoietic stem and progenitor cells and reveal endothelial niches for stem cells. Cell. 121:1109-1121.

38. Kopp, H.G., Avecilla, S.T., Hooper, A.T., and Rafii, S. 2005. The bone marrow vascular niche: home of HSC differentiation and mobilization. Physiology (Bethesda). 20:349-356.

39. Wagers, A.J. 2005. Stem cell grand SLAM. Cell. 121:967-970

40. Jones, D.L., and Fuller, M.T. 2004. Stem cell niche. In Handbook of stem cells. R. Lanza, editor. Elsevier Academic. Boston, Massachusetts, USA. 59-71.

41. Watt, F.M., and Hogan, B.L. 2000. Out of Eden: stem cells and their niches Science. 287:1427-1430.

42. Fuchs, E., Tumbar, T., and Guasch, G. 2004. Socializing with the neighbors: stem cells and their niche. Cell. 116:769-778.

43. Wallenfang, M.R., and Matunis, E. 2003. Developmental biology. Orienting stem cells. Science. 301:1490-1491.

44. Yamashita, Y.M., Jones, D.L., and Fuller, M.T. 2003. Orientation of asymmetric stem cell division by the APC tumor suppressor and centrosome. Science. 301:1547-1550.

45. Morrison, S.J., and Weissman, I.L. 1994. The longterm repopulating subset of hematopoietic stem cells is deterministic and isolatable by phenotype. Immunity. 1:661-673.

46. Christensen, J.L., and Weissman, I.L. 2001. Flk-2 is a marker in hematopoietic stem cell differentiation: a simple method to isolate long-term stem cells. Proc. Natl. Acad. Sci. U. S. A. 98:14541-14546.

47. Passegue, E., Wagers, A.J., Giuriato, S., Anderson, W.C., and Weissman, I.L. 2005. Global analysis of proliferation and cell cycle gene expression in the regulation of hematopoietic stem and progenitor cell fates. J. Exp. Med. 202:1599-1611.

48. El-Badri, N.S., Wang, B.Y., Cherry, and Good, R.A. 1998. Osteoblasts promote engraftment of allogeneic hematopoietic stem cells. Exp. Hematol.
26:110-116.

49. Li, L. 2005. Finding the hematopoietic stem cell niche in the placenta. Dev. Cell. 8:297-298.

50. Abkowitz, J.L., Robinson, A.E., Kale, S., Long, M.W., and Chen, J. 2003. Mobilization of hematopoietic stem cells during homeostasis and after cytokine exposure. Blood. 102:1249-1253.

51. Lapidot, T., Dar, A., and Kollet, O. 2005. How do stem cells find their way home? Blood. 106:1901-1910.

52. Cancelas, J.A., and Williams, D.A. 2006. Stem cell mobilization by beta2-agonists. Nat. Med. 12:278-279.

53. Wright, D.E., Wagers, A.J., Gulati, A.P., Johnson, F.L., and Weissman, I.L. 2001. Physiological migration of hematopoietic stem and progenitor cells. Science. 294:1933-1936.

54. Steidl, U., et al. 2002. Gene expression profiling identifies significant differences between the molecular phenotypes of bone marrow-derived and circulating human CD34+ hematopoietic stem cells. Blood. 99:2037-2044.

55. Adams, G.B., et al. 2006. Stem cell engraftment at the endosteal niche is specified by the calciumsensing receptor. Nature. 439:599-603.

56. Driessen, R.L., Johnston, H.M., and Nilsson, S.K. 2003. Membrane-bound stem cell factor is a key regulator in the initial lodgment of stem cells within the endosteal marrow region. Exp. Hematol. 31:1284-1291.

57. Bernstein, A., Forrester, L., Reith, A.D., Dubreuil, P., and Rottapel, R. 1991. The murine W/c-kit and Steel loci and the control of hematopoiesis. Semin. Hematol. 28:138-142.

58. Li, L., et al. 1998. Human homolog of rat Jagged1 expressed by marrow stroma inhibits differentiation of $32 \mathrm{D}$ cells through interaction with Notch1. Immunity. 8:43-55.

59. Varnum-Finney, B., et al. 2000. Pluripotent, cytokine-dependent, hematopoietic stem cells are immortalized by constitutive Notch 1 signaling. Nat. Med. 6:1278-1281.

60. Reya, T., et al. 2003. A role for Wnt signalling in self-renewal of haematopoietic stem cells. Nature. 423:409-414.

61. Staal, F.J., and Clevers, H.C. 2005. WNT signalling and haematopoiesis: a WNT-WNT situation. Nat. Rev. Immunol. 5:21-30.

62. Bhardwaj, G., et al. 2001. Sonic hedgehog induces the proliferation of primitive human hematopoietic cells via BMP regulation. Nat. Immunol. 2:172-180.

63. de Haan, G., et al. 2003. In vitro generation of longterm repopulating hematopoietic stem cells by fibroblast growth factor-1. Dev Cell. 4:241-251.

64. Jenkins, B.J., Roberts, A.W., Najdovska, M., Grail, D., and Ernst, M. 2005. The threshold of gp130-dependent STAT3 signaling is critical for normal regulation of hematopoiesis. Blood. 105:3512-3520.

65. Rappold, I., et al. 1999. Gp130-signaling synergizes with FL and TPO for the long-term expansion of cord blood progenitors. Leukemia. 13:2036-2048.

66. Nilsson, S.K., et al. 2005. Osteopontin, a key component of the hematopoietic stem cell niche and regulator of primitive hematopoietic progenitor cells. Blood. 106:1232-1239.

67. Stier, S., et al. 2005. Osteopontin is a hematopoietic stem cell niche component that negatively regulates stem cell pool size. J. Exp. Med. 201:1781-1791.

68. Song, X., and Xie, T. 2002. DE-cadherin-mediated cell adhesion is essential for maintaining somatic stem cells in the Drosophila ovary. Proc. Natl. Acad. Sci. U. S. A. 99:14813-14818.

69. Papayannopoulou, T. 2004. Current mechanistic scenarios in hematopoietic stem/progenitor cell mobilization. Blood. 103:1580-1585

70. Wilson, A., et al. 2004. c-Myc controls the balance between hematopoietic stem cell self-renewal and differentiation. Genes Dev. 18:2747-2763.

71. Murphy, M.J., Wilson, A., and Trumpp, A. 2005.
More than just proliferation: Myc function in stem cells. Trends Cell Biol. 15:128-137.

72. Thisse, B., and Thisse, C. 2005. Functions and regulations of fibroblast growth factor signaling during embryonic development. Dev. Biol. 287:390-402.

73. Devine, M.J., Mierisch, C.M., Jang, E., Anderson, P.C., and Balian, G. 2002. Transplanted bone marrow cells localize to fracture callus in a mouse model. J. Orthop. Res. 20:1232-1239.

74. Neiva, K., Sun, Y.X., and Taichman, R.S. 2005. The role of osteoblasts in regulating hematopoietic stem cell activity and tumor metastasis. Braz. J. Med. Biol. Res. 38:1449-1454.

75. Dar, A., et al. 2005. Chemokine receptor CXCR4dependent internalization and resecretion of functional chemokine SDF-1 by bone marrow endothelial and stromal cells. Nat. Immunol. 6:1038-1046.

76. Cancelas, J.A., et al. 2005. Rac GTPases differentially integrate signals regulating hematopoietic stem cell localization. Nat. Med. 11:886-891.

77. Peled, A., et al. 1999. The chemokine SDF-1 stimulates integrin-mediated arrest of CD34(+) cells on vascular endothelium under shear flow. J. Clin. Invest. 104:1199-1211.

78. Kortesidis, A., et al. 2005. Stromal-derived factor-1 promotes the growth, survival, and development of human bone marrow stromal stem cells. Blood. 105:3793-3801.

79. Katayama, Y., et al. 2003. PSGL-1 participates in Eselectin-mediated progenitor homing to bone marrow: evidence for cooperation between E-selectin ligands and alpha4 integrin. Blood. 102:2060-2067.

80. Yang, F.C., et al. 2001. Rac and Cdc42 GTPases control hematopoietic stem cell shape, adhesion, migration, and mobilization. Proc. Natl. Acad. Sci. U. S. A. 98:5614-5618

81. Petit, I., et al. 2002. G-CSF induces stem cell mobilization by decreasing bone marrow SDF-1 and upregulating CXCR4. Nat. Immunol. 3:687-694.

82. McQuibban, G.A., et al. 2001. Matrix metalloproteinase activity inactivates the CXC chemokine stromal cell-derived factor-1. J. Biol. Chem. 276:43503-43508.

83. Semerad, C.L., et al. 2005. G-CSF potently inhibits osteoblast activity and CXCL12 mRNA expression in the bone marrow. Blood. 106:3020-3027.

84. Katayama, Y., et al. 2006. Signals from the sympathetic nervous system regulate hematopoietic stem cell egress from bone marrow. Cell. 124:407-421.

85 . Teitelbaum, S.L. 2000. Bone resorption by osteoclasts. Science. 289:1504-1508.

86. Miyamoto, T., and Suda, T. 2005. Differentiation and function of monocyte/macrophage lineage cells and osteoblasts [In Japanese]. Nippon Rinsho. 63:1511-1516.

87. Lacey, D.L., et al. 1998. Osteoprotegerin ligand is a cytokine that regulates osteoclast differentiation and activation. Cell. 93:165-176.

88. Simonet, W.S., et al. 1997. Osteoprotegerin: a novel secreted protein involved in the regulation of bone density. Cell. 89:309-319.

89. Hirose, J., et al. 2002. A developing picture of lymphopoiesis in bone marrow. Immunol. Rev. 189:28-40

90. Horowitz, M.C., Bothwell, A.L., Hesslein, D.G., Pflugh, D.L., and Schatz, D.G. 2005. B cells and osteoblast and osteoclast development. Immunol. Rev. 208:141-153.

91. Peschon, J.J., et al. 1994. Early lymphocyte expansion is severely impaired in interleukin 7 receptordeficient mice. J. Exp. Med. 180:1955-1960.

92. von Freeden-Jeffry, U., et al. 1995. Lymphopenia in interleukin (IL)-7 gene-deleted mice identifies IL-7 as a nonredundant cytokine. J. Exp. Med. 181:1519-1526.

93. Kincade, P.W., et al. 2002. Lymphoid lineage cells in adult murine bone marrow diverge from those of other blood cells at an early, hormone-sensitive stage. Semin. Immunol. 14:385-394. 